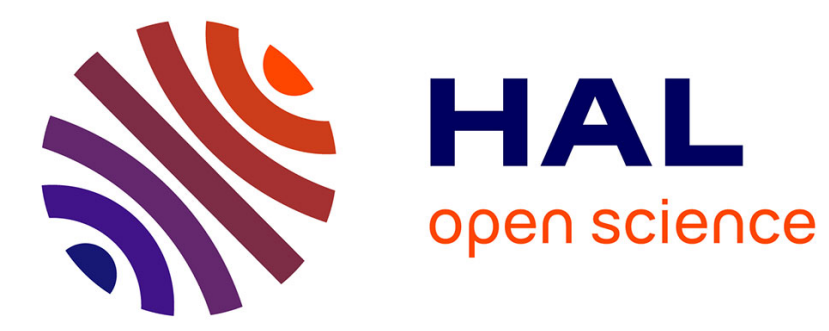

\title{
Existence and approximation for vibro-impact problems with a time-dependent set of constraints
}

\author{
Laetitia Paoli
}

\section{To cite this version:}

Laetitia Paoli. Existence and approximation for vibro-impact problems with a time-dependent set of constraints. Mathematics and Computers in Simulation, 2015, 118, pp.302-309. 10.1016/j.matcom.2014.11.015 . hal-01567478

\section{HAL Id: hal-01567478 \\ https://hal.science/hal-01567478}

Submitted on 24 Jul 2017

HAL is a multi-disciplinary open access archive for the deposit and dissemination of scientific research documents, whether they are published or not. The documents may come from teaching and research institutions in France or abroad, or from public or private research centers.
L'archive ouverte pluridisciplinaire HAL, est destinée au dépôt et à la diffusion de documents scientifiques de niveau recherche, publiés ou non, émanant des établissements d'enseignement et de recherche français ou étrangers, des laboratoires publics ou privés. 


\title{
Existence and approximation for vibro-impact problems with a time-dependent set of constraints
}

\author{
Laetitia Paoli \\ Institut Camille Jordan, UMR CNRS 5208, Université de Saint-Etienne, 23 rue Michelon, 42023 Saint-Etienne Cedex 2, France
}

\begin{abstract}
We consider a discrete mechanical system subjected to perfect time-dependent unilateral constraints, which dynamics is described by a second order measure differential inclusion. The transmission of the velocity at impacts is given by a minimization property of the kinetic energy with respect to the set of kinematically admissible post-impact velocities. We construct a sequence of feasible approximate positions by using a time-stepping algorithm inspired by a kind of Euler discretization of the differential inclusion. We prove the convergence of the approximate trajectories to a solution of the Cauchy problem and we obtain as a by-product a global existence result.
\end{abstract}

Keywords: Discrete mechanical system; Time-dependent constraints; Differential inclusion; Inelastic shocks; Time-stepping scheme

\section{Introduction}

We consider a mechanical system with a finite number of degrees of freedom subjected to frictionless unilateral constraints. If we denote by $u(t)$ its representative point in generalized coordinates and by $K(t)$ the set of admissible positions at any instant $t$, the dynamics of the system is described by a second order differential equation combined with the condition $u(t) \in K(t)$, leading to a measure differential inclusion.

Such problems occur frequently in automotive industry or aeronautics where looseness of joints may create unwanted vibrations and impacts, but they are also of crucial importance in environment for the study of the damages due to earthquakes for instance. One can find an important literature on this topic in the case of time-independent constraints (i.e. when the set of admissible positions does not depend on $t$ ), which is more related to industrial issues, and several existence results have been obtained either by constructing a sequence of approximate trajectories and by proving their convergence to a solution of the Cauchy problem $[24,6,4,5,23,11,20,9,14,22,15,10,7,8,17,19]$, or by using theoretical arguments based on existence results for ordinary differential equations and variational inequalities [1]. On the contrary, very few studies are available in the case of time-dependent constraints, which is more related to environmental issues like earthquakes. In [25] an existence result is established, by considering a generalization of the Yosida-type approximation already proposed in [20]. Unfortunately this technique transforms the differential 
inclusion into a sequence of very stiff ordinary differential equations which are not well suited for implementation (see [21] for a more detailed discussion). More recently another existence result, based on a time-stepping approximation of the problem, has been obtained [2] when the sets $K(t)$ are defined as a finite intersection of complements of convex sets. But this property is a very restrictive assumption and is usually not satisfied.

The aim of this work is thus to propose another time-stepping approximation of the problem and to state its convergence, which will give as a by-product a global existence result, in a more general geometrical setting than in [2]. More precisely we will first derive from the basic mechanical and geometrical description of the problem its mathematical formulation. Then we introduce a time-stepping scheme inspired by some implicit Euler's type discretization and the main steps of the convergence proof are outlined.

\section{Formulation of the problem}

We consider a discrete mechanical system which unconstrained motion is described by the following second order differential equation in $\mathbb{R}^{d}$ :

$$
\ddot{u}=g(t, u) .
$$

We assume that the system is subjected to time-dependent unilateral constraints i.e.

$$
u(t) \in K(t) \quad \forall t
$$

where $K(t)$ is the set of admissible positions at instant $t$, given by the geometrical inequalities

$$
u \in K(t) \Longleftrightarrow f_{\alpha}(t, u) \geq 0, \quad \forall \alpha \in\{1, \ldots, \nu\}, v \geq 1
$$

with smooth functions $f_{\alpha}$. When a contact occurs, i.e. when $u(t) \in \partial K(t)$, a reaction force appears. If we assume that the contact is frictionless then [12]

$$
\ddot{u}-g(t, u)=R \in-N(K(t), u)
$$

where $N(K(t), u)$ is the normal cone to $K(t)$ at $u$ given by

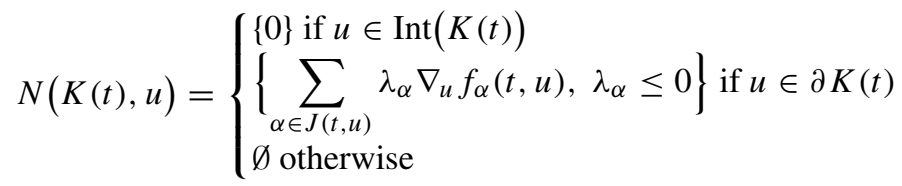

and $J(t, u)=\left\{\alpha \in\{1, \ldots, v\} ; f_{\alpha}(t, u) \leq 0\right\}$ is the set of active constraints at the point $(t, u)$.

As usual for vibro-impact problems, the velocity $\dot{u}$ may be discontinuous and the adequate framework for the solutions is the set of absolutely continuous functions $u$ which derivative $\dot{u}$ belongs to the space of functions of bounded variation. Indeed, if we assume that the right and left velocities exist at some instant $t$, we infer from (2) that

$$
\dot{u}^{+}(t) \in T(t, u(t)), \quad \dot{u}^{-}(t) \in-T(t, u(t))
$$

with

$$
T(t, u)=\left\{v \in \mathbb{R}^{d} ; \partial_{t} f_{\alpha}(t, u)+\left\langle\nabla_{u} f_{\alpha}(t, u), v\right\rangle \geq 0 \text { for all } \alpha \in J(t, u)\right\} .
$$

It follows that $\dot{u}$ may be discontinuous at $t$ if $J(t, u(t)) \neq \emptyset$ and the reaction force $R$ is a measure. Of course the acceleration $\ddot{u}$ should now be understood as the Stieltjes measure $d \dot{u}$ and the measure $d \dot{u}-g(t, u) d t$ should vanish on $\{t ; J(t, u(t))=\emptyset\}$, in order to recover (1) when the constraints are not saturated. More precisely, there exist $v$ scalar measures $\lambda_{\alpha}$ such that

$$
\left\{\begin{array}{l}
d \dot{u}-g(\cdot, u) d t=\sum_{\alpha=1}^{\nu} \lambda_{\alpha} \nabla_{u} f_{\alpha}(\cdot, u) \\
\lambda_{\alpha} \geq 0, \quad \operatorname{Supp}\left(\lambda_{\alpha}\right) \subset\left\{t ; f_{\alpha}(t, u(t))=0\right\} \quad \forall \alpha \in\{1, \ldots, v\} .
\end{array}\right.
$$


Hence, at any contact instant $t$, we obtain

$$
\begin{aligned}
& \dot{u}^{+}(t) \in T(t, u(t)), \quad \dot{u}^{-}(t) \in-T(t, u(t)), \\
& \dot{u}^{+}(t)-\dot{u}^{-}(t) \in-N(K(t), u(t)) .
\end{aligned}
$$

Unfortunately these relations do not define uniquely $\dot{u}^{+}(t)$ and we have to complete the description of the problem by an impact law. We model the transmission of the velocity by assuming that

$$
\dot{u}^{+}(t)=\operatorname{Proj}\left(T(t, u(t)), \dot{u}^{-}(t)\right) \quad \text { if } J(t, u(t)) \neq \emptyset .
$$

Observing that $T(t, u(t))$ is the set of kinematically admissible right velocities at $t$, this relation corresponds to a minimizing property of the kinetic energy which generalizes the definition of standard inelastic shocks introduced by J.J. Moreau [13]. So, for any admissible initial data $\left(u_{0}, v_{0}\right) \in K(0) \times T\left(0, u_{0}\right)$, we will consider the following Cauchy problem:

Problem (P). Find $u:[0, \tau] \rightarrow \mathbb{R}^{d}$, with $\tau>0$, such that

(P1) $u$ is absolutely continuous on $[0, \tau], \dot{u} \in B V\left(0, \tau ; \mathbb{R}^{d}\right)$,

(P2) $u(t) \in K(t)$ for all $t \in[0, \tau]$,

(P3) there exist $v$ scalar measures $\lambda_{\alpha}$ such that

$$
\left\{\begin{array}{l}
d \dot{u}-g(\cdot, u) d t=\sum_{\alpha=1}^{\nu} \lambda_{\alpha} \nabla_{u} f_{\alpha}(\cdot, u) \\
\lambda_{\alpha} \geq 0, \quad \operatorname{Supp}\left(\lambda_{\alpha}\right) \subset\left\{t \in[0, \tau] ; f_{\alpha}(t, u(t))=0\right\} \forall \alpha \in\{1, \ldots, \nu\},
\end{array}\right.
$$

(P4) for all $t \in(0, \tau)$ such that $J(t, u(t)) \neq \emptyset$ we have

$$
\begin{aligned}
\dot{u}^{+}(t) & =\operatorname{Proj}\left(T(t, u(t)), \dot{u}^{-}(t)\right), \\
(\mathrm{P} 5) u(0) & =u_{0}, \dot{u}^{+}(0)=v_{0} .
\end{aligned}
$$

Let us emphasize that the sets $\left\{t \in[0, \tau] ; f_{\alpha}(t, u(t))=0\right\}, \alpha \in\{1, \ldots, v\}$, corresponding to the instants $t$ for which the constraint numbered $\alpha$ is saturated, play the role of free boundaries.

\section{Approximation by a time-stepping scheme}

Let $h>0$ be a given time-step and let $t_{n}=n h$ for all $n \geq 0$. We define

- $U^{-1}=u_{0}-h v_{0}, U^{0}=u_{0}$,

- for all $n \geq 0$

$$
G^{n}=\frac{1}{h} \int_{t_{n}}^{t_{n+1}} g\left(s, U^{n}\right) d s
$$

and

$$
W^{n}=2 U^{n}-U^{n-1}+h^{2} G^{n}, \quad U^{n+1} \in \operatorname{Argmin}_{z \in K\left(t_{n+1}\right)}\left\|W^{n}-z\right\| .
$$

We can observe that, whenever $K\left(t_{n+1}\right)$ is a convex subset of $\mathbb{R}^{d}$, (6) is equivalent to

$$
U^{n+1}=\operatorname{Proj}\left(K\left(t_{n+1}\right), 2 U^{n}-U^{n-1}+h^{2} G^{n}\right) .
$$

Hence this scheme is a natural generalization of the one proposed in [22,15,17]. Furthermore, (5)-(6) may also be interpreted as a generalization of the algorithm proposed in [2] when $K\left(t_{n+1}\right)$ is not convex. Indeed, if we replace in (6) $K\left(t_{n+1}\right)$ by its convex approximation $\tilde{K}\left(t_{n+1}, U^{n}\right)$ defined by

$$
\tilde{K}\left(t_{n+1}, U^{n}\right)=\left\{q \in \mathbb{R}^{d} ; f_{\alpha}\left(t_{n+1}, U^{n}\right)+\left\langle\nabla_{u} f_{\alpha}\left(t_{n+1}, U^{n}\right), q-U^{n}\right\rangle \geq 0 \forall \alpha \in\{1, \ldots, \nu\}\right\}
$$

we obtain

$$
U^{n+1}=\operatorname{Proj}\left(\tilde{K}\left(t_{n+1}, U^{n}\right), 2 U^{n}-U^{n-1}+h^{2} G^{n}\right)
$$


which is equivalent to

$$
U^{n+1}=U^{n}+h V^{n}, \quad V^{n}=\operatorname{Proj}\left(K_{h}\left(t_{n+1}, U^{n}\right), V^{n-1}+h G^{n}\right)
$$

with

$$
K_{h}\left(t_{n+1}, U^{n}\right)=\left\{v \in \mathbb{R}^{d} ; f_{\alpha}\left(t_{n+1}, U^{n}\right)+h\left\langle\nabla_{u} f_{\alpha}\left(t_{n+1}, U^{n}\right), v\right\rangle \geq 0 \forall \alpha \in\{1, \ldots, v\}\right\} .
$$

We recognize here the scheme introduced in [2].

Let us observe that (6) ensures the feasibility of the approximate positions on the contrary of (7), which does not ensure $U^{n} \in K\left(t_{n}\right)$ for all $n \geq 0$ if the functions $f_{\alpha}, \alpha \in\{1, \ldots, v\}$, are not convex with respect to their second argument, i.e. if the sets $K\left(t_{n}\right)$ are not defined as a finite intersection of complements of convex sets.

\section{Convergence result}

For our convergence (and existence) result we adopt the same regularity assumptions for the data as in [2] but we will not assume any convexity property for the functions $f_{\alpha}$. More precisely, let $T>0$. We assume:

(H1) The mappings $f_{\alpha}, \alpha \in\{1, \ldots, v\}$, belong to $C^{2}\left([0, T] \times \mathbb{R}^{d} ; \mathbb{R}\right)$ and for all $t \in[0, T]$, the set $K(t)=\{u \in$ $\left.\mathbb{R}^{d} ; f_{\alpha}(t, u) \geq 0, \forall \alpha \in\{1, \ldots, v\}\right\}$ is not empty.

We define

$$
K=\left\{(t, u) \in[0, T] \times \mathbb{R}^{d} ; u \in K(t)\right\}
$$

and for any $r>0, K_{r}$ is the neighbourhood of $K$ given by

$$
K_{r}=\left\{(s, y) \in[0, T] \times \mathbb{R}^{d} ; \exists(t, u) \in K /|s-t| \leq r,\|y-u\| \leq r\right\} .
$$

(H2) There exist $r>0, m>0$ and $M>0$ such that, for all $(s, y) \in K_{r}$ :

$$
\begin{aligned}
& m \leq\left\|\nabla_{u} f_{\alpha}(s, y)\right\| \leq M, \quad\left|\partial_{t} f_{\alpha}(s, y)\right| \leq M, \\
& \left|\partial_{t}^{2} f_{\alpha}(s, y)\right| \leq M, \quad\left\|\partial_{t} \nabla_{u} f_{\alpha}(s, y)\right\| \leq M, \quad\left\|D_{u}^{2} f_{\alpha}(s, y)\right\| \leq M .
\end{aligned}
$$

(H3) There exist $\gamma>0$ and $\rho>0$ such that, for all $t \in[0, T]$ and for all $u \in K(t)$ :

$$
\sum_{\alpha \in J_{\rho}(t, u)} \lambda_{\alpha}\left\|\nabla_{u} f_{\alpha}(t, u)\right\| \leq \gamma\left\|\sum_{\alpha \in J_{\rho}(t, u)} \lambda_{\alpha} \nabla_{u} f_{\alpha}(t, u)\right\| \quad \forall \lambda_{\alpha} \geq 0, \alpha \in J_{\rho}(t, u)
$$

where

$$
J_{\rho}(t, u)=\left\{\alpha \in\{1, \ldots, v\} ; f_{\alpha}(t, u) \leq \rho\right\} .
$$

Whenever several constraints are saturated at $(t, u)$, this last assumption may be interpreted as a kind of positive linear independence property for the vectors $\left(\nabla_{u} f_{\alpha}(t, u)\right)_{\alpha \in J(t, u)}$ which allows us to consider also cases where the active constraints are not linearly independent. It follows that this geometrical framework is more general than in $[1,15,17,19]$.

(H4) The function $g$ is a Caratheodory function from $[0, T] \times \mathbb{R}^{d}$ with values in $\mathbb{R}^{d}$ and there exist $k_{g}>0$ and $F \in L^{1}(0, T ; \mathbb{R})$ such that, for almost every $t \in[0, T]$ we have

$$
\begin{aligned}
& \|g(t, u)-g(t, \tilde{u})\| \leq k_{g}\|u-\tilde{u}\|, \quad\|g(t, u)\| \leq F(t) \\
& \forall(u, \tilde{u}) \in\left(\mathbb{R}^{d}\right)^{2} \text { such that }(t, u) \in K_{r},(t, \tilde{u}) \in K_{r} .
\end{aligned}
$$

As a consequence of these assumptions we obtain

Proposition 1 ([18]). For all $n \in\{0, \ldots,\lfloor T / h\rfloor-1\}$, there exists a family of non negative real numbers $\left(\lambda_{\alpha}^{n}\right)_{\alpha \in\{1, \ldots, \nu\}}$ such that $\lambda_{\alpha}^{n}=0$ for all $\alpha \notin J\left(t_{n+1}, U^{n+1}\right)$ and

$$
V^{n}-V^{n-1}-h G^{n}=\sum_{\alpha=1}^{\nu} \lambda_{\alpha}^{n} \nabla_{u} f_{\alpha}\left(t_{n+1}, U^{n+1}\right) .
$$


This relation is the discrete analogous of property (P3). Furthermore it can be reformulated as

$$
\frac{V^{n}-V^{n-1}}{h}-G^{n} \in-N\left(K\left(t_{n+1}\right), U^{n+1}\right) \quad \forall n \in\{0, \ldots,\lfloor T / h\rfloor-1\}
$$

which is a kind of Euler discretization of the measure differential inclusion (3).

This is not enough to ensure the good behaviour of the algorithm at impacts. Indeed, when several constraints may be saturated at the same instant $t$, continuity on data does not hold in general. The study of the model problem of a material point moving in an angular domain of $\mathbb{R}^{2}$ shows that a small change in the initial position can lead to a completely different trajectory after an impact if the edge angle is obtuse. From a computational point of view, this bad property may create a kind of numerical unpredictability, due to round-up errors, and should be avoided.

In the case of time-independent constraints, it has been proved in $[1,16]$ that a necessary and sufficient condition to ensure continuity on data is given by

$$
\left\langle\nabla_{u} f_{\alpha}(t, u(t)), \nabla_{u} f_{\beta}(t, u(t))\right\rangle \leq 0 \quad \forall(\alpha, \beta) \in J(t, u(t))^{2}, \alpha \neq \beta
$$

i.e. the active constraints along the trajectory create right or acute angles. Hence we will also consider a similar geometrical assumption in our convergence result.

We define the sequence of approximate velocities $\left(v_{h}\right)_{h>0}$ by

$$
\begin{aligned}
& v_{h}(t)=V^{n}=\frac{U^{n+1}-U^{n}}{h} \quad \forall t \in[n h,(n+1) h), \forall n \in\{0, \ldots, N(h)-1\}, \\
& v_{h}(t)=V^{N(h)-1} \quad \forall t \in[N(h) h, T],
\end{aligned}
$$

with $N(h)=\left\lfloor\frac{T}{h}\right\rfloor$. Let us define also the sequence of approximate trajectories $\left(u_{h}\right)_{h>0}$ by a linear interpolation of the $U^{n}$ 's i.e.

$$
\begin{aligned}
& u_{h}(t)=U^{n}+(t-n h) V^{n} \quad \forall t \in[n h,(n+1) h], \forall n \in\{0, \ldots, N(h)-1\}, \\
& u_{h}(t)=U^{N(h)}+(t-N(h) h) V^{N(h)-1} \quad \forall t \in[N(h) h, T] .
\end{aligned}
$$

We obtain

Theorem 2 ([18]). Let us assume that $(\mathrm{H} 1)-(\mathrm{H} 4)$ hold. Let $\left(u_{0}, v_{0}\right) \in K(0) \times T\left(0, u_{0}\right)$. Then, we can extract subsequences, still denoted $\left(u_{h}\right)_{h>0}$ and $\left(v_{h}\right)_{h>0}$, and there exist $u \in C^{0}\left([0, T] ; \mathbb{R}^{d}\right)$ and $v \in B V\left(0, T ; \mathbb{R}^{d}\right)$ such that

$$
\begin{aligned}
& u_{h} \rightarrow u \quad \text { strongly in } C^{0}\left([0, T] ; \mathbb{R}^{d}\right), \\
& v_{h} \rightarrow v \text { pointwise in }[0, T], \\
& d v_{h} \rightarrow d v \quad \text { weakly* in } \mathcal{M}^{1}\left(0, T ; \mathbb{R}^{d}\right) .
\end{aligned}
$$

\section{Moreover}

$$
u(t)=u_{0}+\int_{0}^{t} v(s) d s \quad \forall t \in[0, T]
$$

and $u$ satisfies properties $(\mathrm{P} 1)-(\mathrm{P} 3)$. Furthermore, if for all $t \in[0, T]$, we have

$$
\left\langle\nabla_{u} f_{\alpha}(t, u(t)), \nabla_{u} f_{\beta}(t, u(t))\right\rangle \leq 0 \quad \forall(\alpha, \beta) \in J(t, u(t))^{2}, \alpha \neq \beta,
$$

then $u$ satisfies also properties (P4) and (P5) and is a solution of Problem (P) on $[0, T]$.

Let us recall that uniqueness is not true in general $[3,24,1]$. Hence, we cannot expect the convergence of the whole sequence of approximate solutions.

As usual for this kind of problems, the proof is divided in several steps. First we establish some a priori estimates for the sequence $\left(v_{h}\right)_{h>0}$ in $L^{\infty}\left(0, T ; \mathbb{R}^{d}\right)$ and in $B V\left(0, T ; \mathbb{R}^{d}\right)$. By applying Ascoli's and Helly's theorems we may extract a subsequence, still denoted $\left(u_{h}\right)_{h>0}$ and $\left(v_{h}\right)_{h>0}$, such that

$$
\begin{aligned}
& u_{h} \rightarrow u \quad \text { strongly in } C^{0}\left([0, T] ; \mathbb{R}^{d}\right), \\
& v_{h} \rightarrow v \quad \text { pointwise in }[0, T], \\
& d v_{h} \rightarrow d v \quad \text { weakly* in } \mathcal{M}^{1}\left(0, T ; \mathbb{R}^{d}\right) .
\end{aligned}
$$




\section{Moreover}

$$
u(t)=u_{0}+\int_{0}^{t} v(s) d s \quad \forall t \in[0, T]
$$

and we prove that the limit trajectory is feasible, i.e. $u(t) \in K(t)$ for all $t \in[0, T]$. Then using Proposition 1 , we have

$$
\begin{aligned}
d v_{h} & =\sum_{n=1}^{N(h)-1}\left(V^{n}-V^{n-1}\right) \delta(t-n h) \\
& =\sum_{n=1}^{N(h)-1} h G^{n} \delta(t-n h)+\sum_{n=1}^{N(h)-1} \sum_{\alpha=1}^{\nu} \lambda_{\alpha}^{n} \nabla_{u} f_{\alpha}\left(t_{n+1}, U^{n+1}\right) \delta(t-n h)
\end{aligned}
$$

with

$$
\lambda_{\alpha}^{n} \geq 0 \quad \forall \alpha \in\{1, \ldots, v\}, \quad \lambda_{\alpha}^{n}=0 \quad \forall \alpha \notin J\left(t_{n+1}, U^{n+1}\right)
$$

for all $n \in\{0, \ldots, N(h)-1\}$. By observing that

$$
\left\|\sum_{\alpha=1}^{v} \lambda_{\alpha}^{n} \nabla_{u} f_{\alpha}\left(t_{n+1}, U^{n+1}\right)\right\| \leq\left\|V^{n}-V^{n-1}\right\|+h\left\|G^{n}\right\| \quad \forall n \in\{1, \ldots, N(h)-1\}
$$

we obtain

$$
\sum_{n=1}^{N(h)-1}\left|\lambda_{\alpha}^{n}\right| \leq \frac{\gamma}{m}\left(T V\left(v_{h}\right)+\|F\|_{L^{1}\left(0, T ; \mathbb{R}^{d}\right)}\right) \quad \forall \alpha \in\{1, \ldots, v\} .
$$

Next we define

$$
\lambda_{\alpha, h}(t)=\sum_{n=1}^{N(h)-1} \lambda_{\alpha}^{n} \delta(t-n h), \quad \lambda_{\alpha}^{n} \geq 0, \forall \alpha \in\{1, \ldots, v\} .
$$

Possibly extracting another subsequence, there exist non negative scalar measures $\lambda_{\alpha}$ such that, for all $\alpha \in\{1, \ldots, \nu\}$,

$$
\lambda_{\alpha, h} \rightarrow \lambda_{\alpha} \quad \text { weakly* in } \mathcal{M}^{1}(0, T ; \mathbb{R})
$$

and we get

$$
d \dot{u}=d v=\sum_{\alpha=1}^{v} \lambda_{\alpha} \nabla_{u} f_{\alpha}(\cdot, u)+g(\cdot, u) d t .
$$

Furthermore for all $\alpha \in\{1, \ldots, v\}$ we have

$$
\operatorname{Supp}\left(\lambda_{\alpha}\right) \subset\left\{t \in[0, T] ; f_{\alpha}(t, u(t))=0\right\} .
$$

Finally there remains to study the transmission of the velocities at impacts.

Let $t \in[0, T)$. We already know that

$$
v^{+}(t) \in T(t, u(t)), \quad v^{-}(t) \in-T(t, u(t))
$$

and

$$
v^{+}(t)-v^{-}(t) \in-N(K(t), u(t))=\sum_{\alpha \in J(t, u(t))} \mathbb{R}^{+} \nabla_{u} f_{\alpha}(t, u(t)) .
$$

We have to prove that

$$
v^{+}(t)=\operatorname{Proj}\left(T(t, u(t)), v^{-}(t)\right) \quad \text { if } J(t, u(t)) \neq \emptyset .
$$

Let us assume that $J(t, u(t)) \neq \emptyset$. We split $J(t, u(t))$ as

$$
J(t, u(t))=J_{1}(t, u(t)) \cup J_{2}(t, u(t))
$$


with

$$
\begin{aligned}
J_{1}(t, u(t))= & \left\{\alpha \in J(t, u(t)) ; \exists r_{\alpha}>0, \exists h_{\alpha} \in\left(0, h_{1}\right] / \forall h \in\left(0, h_{\alpha}\right],\right. \\
& \left.\forall n h \in\left[t-\frac{r_{\alpha}}{4(C+1)}, t+\frac{r_{\alpha}}{4(C+1)}\right] \cap[0, T], f_{\alpha}\left(t_{n+1}, U^{n+1}\right)>0\right\}
\end{aligned}
$$

and

$$
\begin{aligned}
J_{2}(t, u(t))= & \left\{\alpha \in J(t, u(t)) ; \forall r_{\alpha}>0, \forall h_{\alpha} \in\left(0, h_{1}\right], \exists h \in\left(0, h_{\alpha}\right],\right. \\
& \left.\exists n h \in\left[t-\frac{r_{\alpha}}{4(C+1)}, t+\frac{r_{\alpha}}{4(C+1)}\right] \cap[0, T] / f_{\alpha}\left(t_{n+1}, U^{n+1}\right) \leq 0\right\} .
\end{aligned}
$$

We infer that there exists a neighbourhood $\mathcal{V}(t)$ of the instant $t$ such that, for all $h$ small enough

$$
J\left(t_{n+1}, U^{n+1}\right) \subset J_{2}(t, u(t)) \quad \forall n h \in \mathcal{V}(t)
$$

i.e.

$$
V^{n}-V^{n-1}=h G^{n}+\sum_{\alpha \in J_{2}(t, u(t))} \lambda_{\alpha}^{n} \nabla_{u} f_{\alpha}\left(t_{n+1}, U^{n+1}\right) \quad \forall n h \in \mathcal{V}(t) .
$$

It follows that

$$
v^{+}(t)-v^{-}(t)=\sum_{\alpha \in J_{2}(t, u(t))} \bar{\lambda}_{\alpha} \nabla_{u} f_{\alpha}(t, u(t)), \quad \bar{\lambda}_{\alpha} \geq 0
$$

and we have to prove that, for all $w \in T(t, u(t))$

$$
\left\langle v^{-}(t)-v^{+}(t), w-v^{+}(t)\right\rangle=-\sum_{\alpha \in J_{2}(t, u(t))} \bar{\lambda}_{\alpha}\left\langle\nabla_{u} f_{\alpha}(t, u(t)), w-v^{+}(t)\right\rangle \leq 0 .
$$

But, for all $\alpha \in J_{2}(t, u(t))$, we have

$$
\begin{aligned}
\left\langle\nabla_{u} f_{\alpha}(t, u(t)), w-v^{+}(t)\right\rangle= & (\overbrace{\partial_{t} f_{\alpha}(t, u(t))+\left\langle\nabla_{u} f_{\alpha}(t, u(t)), w\right\rangle}^{\geq 0}) \\
& -\left(\partial_{t} f_{\alpha}(t, u(t))+\left\langle\nabla_{u} f_{\alpha}(t, u(t)), v^{+}(t)\right\rangle\right) .
\end{aligned}
$$

It follows that

$$
\left\langle v^{-}(t)-v^{+}(t), w-v^{+}(t)\right\rangle \leq \sum_{\alpha \in J_{2}(t, u(t))} \bar{\lambda}_{\alpha}\left(\partial_{t} f_{\alpha}(t, u(t))+\left\langle\nabla_{u} f_{\alpha}(t, u(t)), v^{+}(t)\right\rangle\right)
$$

and we prove that

$$
\partial_{t} f_{\alpha}(t, u(t))+\left\langle\nabla_{u} f_{\alpha}(t, u(t)), v^{+}(t)\right\rangle=0 \quad \alpha \in J_{2}(t, u(t))
$$

which allows us to conclude.

\section{Conclusion}

Starting from the mechanical description of the dynamics of discrete mechanical systems subjected to frictionless time-dependent unilateral constraints, we have derived a mathematical formulation of the problem in terms of measure differential inclusions. Then feasible approximate solutions are built by using a time-stepping scheme inspired by implicit Euler's methods. Finally a convergence result is stated, leading also to a global existence result, and the main steps of the proof are outlined. 


\section{References}

[1] P. Ballard, The dynamics of discrete mechanical systems with perfect unilateral constraints, Arch. Ration. Mech. Anal. 154 (2000) $199-274$.

[2] F. Bernicot, A. Lefebvre-Lepot, Existence results for nonsmooth second-order differential inclusions, convergence result for a numerical scheme and application to the modelling of inelastic collisions, Confluentes Math. 2 (4) (2010) 445-471.

[3] A. Bressan, Questioni di regolarita e di unicita del moto in presenza di vincoli olonomi unilaterali, Rend. Semin. Mat. Univ. Padova 29 (1959) 271-315.

[4] G. Buttazzo, D. Percivale, Sull'approssimazione del problema del rimbalzo unidimensionale, Ric. Mat. 30 (1981) $217-231$.

[5] G. Buttazzo, D. Percivale, On the approximation of the elastic bounce problem on Riemannian manifolds, J. Differential Equations 47 (1983) $227-245$.

[6] M. Carriero, E. Pascali, Il problema del rimbalzo unidimensionale e sue approssimazioni con penalizzazioni non convesse, Rend. Mat. VI. Ser. 13 (1980) 541-553.

[7] R. Dzonou, M.D.P. Monteiro Marques, Sweeping process for inelastic impact problem with a general inertia operator, Eur. J. Mech. A Solids 26 (2007) 474-490.

[8] R. Dzonou, M.D.P. Monteiro Marques, L. Paoli, A convergence result for a vibro-impact problem with a general inertia operator, Nonlinear Dynam. 58 (1/2) (2009) 361-384.

[9] M. Mabrouk, A unified variational model for the dynamics of perfect unilateral constraints, Eur. J. Mech. A Solids 17 (1998) 819-842.

[10] B. Maury, A time-stepping scheme for inelastic collisions, numerical handling of the nonoverlapping constraint, Numer. Math. 102 (2006) 649-679.

[11] M.D.P. Monteiro Marques, Differential Inclusions in Nonsmooth Mechanical Problems, Birkhauser, Boston, 1993.

[12] J.J. Moreau, Les liaisons unilatérales et le principe de Gauss, C.R. Acad. Sci., Paris 256 (1963) 871-874.

[13] J.J. Moreau, Standard inelastic shocks and the dynamics of unilateral constraints, in: G. Del Piero, F. Maceri (Eds.), Unilateral Problems in Structural Analysis, in: CISM Courses and Lectures, no. 288, Springer-Verlag, 1985, pp. 173-221.

[14] L. Paoli, An existence result for vibrations with unilateral constraints: case of a nonsmooth set of constraints, Math. Models Methods Appl. Sci. 10 (6) (2000) 815-831.

[15] L. Paoli, An existence result for non-smooth vibro-impact problems, J. Differential Equations 211 (2005) 247-281.

[16] L. Paoli, Continuous dependence on data for vibro-impact problems, Math. Models Methods Appl. Sci. 15 (1) (2005) 53-93.

[17] L. Paoli, Time discretization of rigid body dynamics with perfect unilateral constraints I and II, Arch. Ration. Mech. Anal. 198 (2010) 457-503. and 505-568.

[18] L. Paoli, A position-based time-stepping algorithm for vibro-impact problems with a moving set of constraints, Confluentes Math. 3 (2) (2011) 263-290.

[19] L. Paoli, A proximal-like algorithm for vibro-impact problems with a non smooth set of constraints, J. Differential Equations 250 (2011) $476-514$.

[20] L. Paoli, M. Schatzman, Mouvement à un nombre fini de degrés de liberté avec contraintes unilatérales: cas avec perte d'énergie, Mod. Math. Anal. Num. 27 (1993) 673-717.

[21] L. Paoli, M. Schatzman, Ill-posedness in vibro-impact and its numerical consequences, in: Proceedings of European Congress on COmputational Methods in Applied Sciences and engineering (ECCOMAS), CD Rom, 2000.

[22] L. Paoli, M. Schatzman, A numerical scheme for impact problems I and II, SIAM J. Numer. Anal. 40 (2) (2002) 702-733 and $734-768$.

[23] D. Percivale, Bounce problem with weak hypotheses of regularity, Ann. Mat. Pura Appl. (4) 143 (1986) $259-274$.

[24] M. Schatzman, A class of nonlinear differential equations of second order in time, Nonlinear Anal. TMA 2 (1978) 355-373.

[25] M. Schatzman, Penalty method for impact in generalized coordinates, Phil. Trans. R. Soc. A 359 (2001) 2429-2446. 